\title{
DR2003055
}

$\mathrm{SiO} 2 \mathrm{Al} 2 \mathrm{O} 3 \mathrm{Fe} 2 \mathrm{O} 3 \mathrm{FeO} \mathrm{MnO} \mathrm{MgO} \mathrm{CaO} \mathrm{Na} 2 \mathrm{O}$ K2O TiO2 P2O5 Total

Pitcairn

Small Volcanoes

$\begin{array}{lll}\text { PNDR1-1 } & 57.4 & 17.0 \\ \text { PNDR1-2 } & 58.8 & 16.8 \\ \text { PNDR1-3 } & 58.8 & 16.9 \\ \text { PNDR1-4 } & 58.6 & 17.1 \\ \text { PNDR2-1 } & 50.9 & 14.8 \\ \text { PNDR3-1 } & 51.5 & 16.9 \\ \text { PNDR3-2 } & 51.0 & 16.6 \\ \text { PNDR3-4 } & 51.2 & 16.7 \\ \text { PNDR3-5 } & 51.9 & 17.5 \\ \text { PNDR3-6 } & 53.0 & 17.0 \\ \text { PNDR3-7 } & 51.3 & 16.7 \\ \text { PNDR5-1 } & 50.8 & 16.6 \\ \text { PNDR5-2 } & 51.9 & 17.4 \\ \text { PNDR11-2 } & 58.0 & 16.6 \\ \text { PNDR11-4 } & 59.2 & 17.3 \\ \text { PNDR11-5 } & 59.4 & 17.4 \\ \text { PNDR11-6 } & 59.2 & 17.5 \\ \text { PNDR11-7 } & 61.6 & 17.9 \\ \text { PNDR12-1 } & 55.9 & 16.2 \\ \text { PNDR12-2 } & 55.6 & 16.2 \\ \text { PNDR12-3 } & 56.3 & 16.8 \\ \text { PNDR12-4 } & 56.5 & 16.8 \\ \text { PNDR12-7 } & 56.6 & 16.7 \\ \text { PN8-1 } & 52.5 & 15.8 \\ \text { PN8-4 } & 58.9 & 18.3 \\ \text { PN8-7 } & 54.3 & 16.4 \\ \text { PN8-10 } & 53.0 & 15.7 \\ \text { Parasite Cones } & & \\ \text { PNDR13-2 } & 58.5 & 17.8 \\ \text { PNDR13-4 } & 56.8 & 17.1 \\ \text { PNDR13-5 } & 60.0 & 18.1 \\ \text { PNDR13-6 } & 58.1 & 17.9 \\ \text { PNDR13-7 } & 57.2 & 17.2 \\ \text { PN13-5 } & 60.0 & 18.1 \\ \text { PN13-11 } & 58.9 & 17.0 \\ \text { PN13-14 } & & 17.1\end{array}$

$\begin{array}{rrrrrrrrr}5.4 & 0.19 & 0.41 & 2.1 & 5.9 & 5.1 & 0.31 & 0.19 & 94.3 \\ 5.4 & 0.19 & 0.39 & 2.0 & 5.8 & 5.2 & 0.33 & 0.17 & 95.4 \\ 5.4 & 0.19 & 0.41 & 2.1 & 5.9 & 5.1 & 0.33 & 0.19 & 95.5 \\ 5.4 & 0.19 & 0.41 & 2.2 & 5.8 & 5.0 & 0.33 & 0.18 & 95.7 \\ 9.6 & 0.16 & 3.31 & 6.8 & 4.6 & 2.2 & 1.79 & 1.36 & 95.9 \\ 8.8 & 0.20 & 2.22 & 4.9 & 5.2 & 3.0 & 1.08 & 1.07 & 95.3 \\ 9.0 & 0.22 & 2.35 & 4.9 & 5.4 & 3.0 & 1.13 & 1.22 & 95.3 \\ 9.0 & 0.21 & 2.26 & 4.9 & 5.4 & 3.0 & 1.13 & 1.09 & 95.3 \\ 9.3 & 0.19 & 2.36 & 5.2 & 4.7 & 3.2 & 1.77 & 0.64 & 97.1 \\ 8.0 & 0.19 & 1.84 & 4.5 & 5.4 & 3.4 & 0.95 & 0.81 & 95.5 \\ 8.9 & 0.23 & 2.27 & 4.9 & 5.4 & 3.1 & 1.13 & 1.09 & 95.5 \\ 9.3 & 0.21 & 2.28 & 5.0 & 5.7 & 2.8 & 1.14 & 1.11 & 95.5 \\ 9.6 & 0.22 & 2.25 & 5.1 & 4.9 & 3.2 & 1.68 & 0.59 & 97.0 \\ 6.3 & 0.24 & 0.12 & 2.0 & 6.6 & 5.0 & 0.17 & 0.13 & 95.5 \\ 7.0 & 0.22 & 0.13 & 2.1 & 5.7 & 5.2 & 0.28 & 0.07 & 97.5 \\ 6.4 & 0.25 & 0.10 & 1.9 & 5.8 & 5.3 & 0.22 & 0.06 & 97.0 \\ 6.4 & 0.22 & 0.10 & 1.9 & 5.8 & 5.3 & 0.21 & 0.06 & 96.9 \\ 6.6 & 0.23 & 0.12 & 2.1 & 6.0 & 5.3 & 0.23 & 0.13 & 100.6 \\ 7.6 & 0.23 & 1.06 & 3.6 & 5.4 & 3.5 & 0.67 & 0.49 & 95.0 \\ 8.1 & 0.23 & 1.20 & 3.8 & 5.4 & 3.6 & 0.72 & 0.56 & 95.7 \\ 8.8 & 0.22 & 1.33 & 4.0 & 4.6 & 3.6 & 1.20 & 0.35 & 97.4 \\ 8.8 & 0.22 & 1.34 & 4.0 & 4.7 & 3.6 & 1.21 & 0.35 & 97.7 \\ 8.8 & 0.22 & 1.33 & 4.0 & 4.6 & 3.6 & 1.20 & 0.36 & 97.6 \\ 8.4 & 0.19 & 2.53 & 5.1 & 5.0 & 2.7 & 1.20 & 1.16 & 94.9 \\ 4.8 & 0.17 & 1.32 & 2.0 & 5.0 & 6.1 & 0.85 & 0.33 & 98.1 \\ 9.1 & 0.19 & 2.68 & 5.3 & 4.4 & 2.8 & 1.95 & 0.69 & 97.9 \\ 8.7 & 0.19 & 2.62 & 5.0 & 5.0 & 2.7 & 1.22 & 1.24 & 95.7 \\ & & & & & & & & \\ 6.8 & 0.19 & 0.79 & 2.9 & 5.1 & 4.6 & 0.73 & 0.22 & 97.8 \\ 6.5 & 0.20 & 0.76 & 2.7 & 6.0 & 4.4 & 0.46 & 0.35 & 95.7 \\ 7.2 & 0.21 & 0.85 & 2.9 & 5.4 & 4.5 & 0.78 & 0.39 & 100.5 \\ 7.1 & 0.19 & 0.84 & 2.9 & 5.1 & 4.5 & 0.75 & 0.21 & 97.9 \\ 6.4 & 0.20 & 0.78 & 2.8 & 5.8 & 4.4 & 0.45 & 0.36 & 95.9 \\ 7.2 & 0.21 & 0.85 & 2.9 & 5.4 & 4.5 & 0.78 & 0.39 & 100.5 \\ 5.5 & 0.18 & 0.41 & 2.0 & 6.1 & 5.0 & 0.31 & 0.21 & 95.9 \\ 5.5 & 0.18 & 0.42 & 2.0 & 6.1 & 5.0 & 0.31 & 0.21 & 95.8\end{array}$




\section{Large Volcanoes}

Adams

PNDR6-1

PNDR6-3

PNDR6-4

PNDR6-5

PNDR6-6

PNDR6-7

PNDR14-2

PNDR14-3

PNDR14-4

PN1-1

PN1-2

PN1-3

PN1-5

PN1-6

PN2-1

PN2-4

PN2-5

PN9-1

PN9-7

Bounty

PN5-4

PN12-2

PN11-1

PN11-2

PN11-4

PN14-13

PN14-8

PN14-6

PN14-1

PN3-11

PN3-8

PN3-7

PN3-6

PN3-5

PN3-4

Societies

Small Volcanoes

Volcano 4

PN16-1

PN16-2

PN16-4

PN16-5

PN16-9

PN16-10

PN16-11

PN16-12

DTH04-01*

\begin{tabular}{|c|c|c|c|c|c|c|c|c|c|c|}
\hline $\mathrm{O2}$ & $\mathrm{Al} 2 \mathrm{O} 3 \mathrm{Fe}$ & $\mathrm{FeO}$ & $\mathrm{MnO}$ & $\mathrm{MgO}$ & $\mathrm{CaO}$ & 1 & K2O & io & & \\
\hline 48.3 & 15.8 & 10.3 & 0.17 & 6.30 & 10.0 & 3.3 & 1.0 & 3.01 & 0.36 & 98.7 \\
\hline 48.7 & 16.0 & 10.5 & 0.17 & 6.38 & 10.2 & 2.3 & 1.2 & 3.03 & 0.36 & 99.1 \\
\hline 47.6 & 15.1 & 9.7 & 0.18 & 5.90 & 9.6 & 3.6 & 0.9 & 1.85 & 0.61 & 95.4 \\
\hline 47.7 & 15.1 & 9.6 & 0.17 & 5.88 & 9.3 & 3.8 & 0.9 & 1.86 & 0.62 & 95.3 \\
\hline 47.8 & 15.7 & 10.2 & 0.16 & 6.24 & 10.0 & 3.3 & 1.0 & 2.96 & .35 & 97.9 \\
\hline 48.3 & 15.7 & 10.2 & 0.16 & 6.28 & 10.0 & 3.3 & 1.0 & 3.00 & 0.37 & 98.5 \\
\hline 48.8 & 15.0 & 10.1 & 0.16 & 5.61 & 9.9 & 3.3 & 1.2 & 3.45 & 0.41 & 98.1 \\
\hline 47.9 & 14.5 & 9.6 & 0.17 & 5.29 & 9.5 & 3.6 & 1.2 & 2.10 & 0.73 & 94.9 \\
\hline 51.0 & 15.5 & 9.6 & 0.19 & 5.87 & 9.9 & 3.3 & 1.2 & 3.58 & 0.74 & 101.0 \\
\hline 61.0 & 17.5 & 6.2 & 0.19 & 0.50 & 1.9 & 5.5 & 4.8 & 0.47 & 0.14 & 98.4 \\
\hline 51.0 & 14.3 & 11.7 & 0.18 & 4.35 & 8.4 & 1.7 & 1.6 & 3.77 & 0.78 & 98.2 \\
\hline 48.9 & 14.6 & 10.4 & 0.16 & 6.22 & 10.8 & 2.4 & 0.9 & 2.88 & 0.48 & 98.1 \\
\hline 50.4 & 14.2 & 12.1 & 0.20 & 4.88 & 9.2 & 1.9 & 1.3 & 3.64 & 0.68 & 98.9 \\
\hline 50.0 & 15.3 & 9.6 & 0.16 & 5.90 & 9.1 & 3.4 & 0.9 & 3.11 & 0.34 & 98.2 \\
\hline 53.8 & 15.9 & 10.1 & 0.18 & 3.59 & 7.0 & 4.0 & 2.4 & 2.96 & 1.42 & 101.8 \\
\hline 47.8 & 15.9 & 9.9 & 0.16 & 5.76 & 9.4 & 3.6 & 1.5 & 3.35 & 0.48 & 98.1 \\
\hline 48.1 & 15.7 & 10.1 & 0.15 & 6.51 & 9.4 & 2.4 & 1.5 & 3.26 & 0.75 & 98.2 \\
\hline 49.4 & 14.8 & 10.4 & 0.18 & 6.60 & 11.4 & 2.5 & 0.8 & 2.73 & 0.47 & 99.7 \\
\hline 49.2 & 14.7 & 10.4 & 0.17 & 6.63 & 11.2 & 2.5 & 0.8 & 2.78 & 0.48 & 99.2 \\
\hline
\end{tabular}

$\begin{array}{ll}54.3 & 14.9 \\ 52.4 & 14.7 \\ 48.7 & 15.8 \\ 48.2 & 15.8 \\ 49.2 & 15.3 \\ 49.3 & 14.9 \\ 48.5 & 15.4 \\ 49.7 & 14.8 \\ 49.3 & 14.8 \\ 49.3 & 15.7 \\ 47.8 & 16.3 \\ 49.8 & 16.4 \\ 48.1 & 15.9 \\ 48.0 & 15.8 \\ 48.5 & 15.8\end{array}$

$\begin{array}{llll}1.2 & 0.21 & 3.57 & 6\end{array}$ $\begin{array}{llll}10.9 & 0.18 & 3.70 & 6.8\end{array}$ $\begin{array}{llll}10.0 & 0.13 & 5.07 & 8.6\end{array}$

$\begin{array}{llll}9.7 & 0.16 & 5.08 & 8.6\end{array}$

$\begin{array}{llll}9.9 & 0.17 & 4.01 & 8.0\end{array}$

$\begin{array}{llll}9.7 & 0.17 & 4.06 & 7.5\end{array}$

$\begin{array}{llll}9.5 & 0.15 & 5.13 & 8.1\end{array}$

$\begin{array}{llll}9.2 & 0.16 & 5.39 & 8.7\end{array}$

$\begin{array}{llll}10.2 & 0.17 & 4.33 & 3.6\end{array}$

$\begin{array}{llll}9.9 & 0.16 & 4.87 & 9.3\end{array}$

$\begin{array}{llll}9.7 & 0.15 & 5.27 & 8.8\end{array}$

$\begin{array}{llll}10.0 & 0.17 & 5.47 & 10.2\end{array}$

$\begin{array}{llll}9.9 & 0.15 & 5.43 & 10.3\end{array}$

$\begin{array}{llll}10.0 & 0.17 & 5.57 & 10.3\end{array}$

$\begin{array}{lllll}4.1 & 2.2 & 3.24 & 1.12 & 101.9\end{array}$

$\begin{array}{lllll}1.5 & 2.0 & 3.03 & 0.92 & 96.4\end{array}$

$\begin{array}{lllll}2.0 & 1.8 & 4.00 & 0.81 & 97.0\end{array}$

$\begin{array}{lllll}1.9 & 1.7 & 3.99 & 0.80 & 96.3\end{array}$

$\begin{array}{lllll}4.1 & 2.2 & 3.90 & 0.71 & 97.7\end{array}$

$\begin{array}{lllll}4.4 & 1.6 & 2.17 & 0.91 & 95.2\end{array}$

$\begin{array}{lllll}4.2 & 1.3 & 2.12 & 0.76 & 95.6\end{array}$

$\begin{array}{lllll}3.9 & 0.9 & 1.95 & 0.57 & 95.6\end{array}$

$\begin{array}{lllll}1.7 & 1.8 & 3.62 & 0.76 & 95.3\end{array}$

$\begin{array}{lllll}3.9 & 1.6 & 3.51 & 0.49 & 99.1\end{array}$

$\begin{array}{lllll}2.0 & 1.7 & 3.45 & 0.67 & 96.3\end{array}$

$\begin{array}{llllll}3.8 & 1.4 & 3.17 & 0.75 & 101.6\end{array}$

$\begin{array}{lllll}3.7 & 1.4 & 3.12 & 0.42 & 98.8\end{array}$

$\begin{array}{lllll}2.2 & 1.4 & 3.10 & 0.56 & 97.3\end{array}$ $\begin{array}{lllllllll}10.1 & 0.16 & 5.15 & 9.6 & 3.9 & 1.4 & 3.17 & 0.43 & 98.7\end{array}$

$\begin{array}{ll}62.3 & 18.2 \\ 59.3 & 17.8 \\ 62.6 & 18.0 \\ 63.8 & 18.9 \\ 63.3 & 18.9 \\ 63.2 & 19.0 \\ 63.6 & 18.9 \\ 63.8 & 18.9 \\ 61.5 & 18.0\end{array}$

$\begin{array}{llll}5.5 & 0.17 & 0.42 & 1.8 \\ 6.7 & 0.20 & 0.65 & 2.1 \\ 5.8 & 0.19 & 0.44 & 2.2 \\ 3.1 & 0.20 & 0.74 & 1.1 \\ 3.2 & 0.20 & 0.75 & 1.1 \\ 3.3 & 0.19 & 0.78 & 1.2 \\ 2.9 & 0.20 & 0.66 & 1.1 \\ 3.0 & 0.18 & 0.67 & 1.1 \\ 0.0 & 0.17 & 0.89 & 1.4\end{array}$

$\begin{array}{lll}5.0 & 5.6 & 0.30 \\ 4.8 & 5.5 & 0.38\end{array}$

$\begin{array}{lllll}5.0 & 5.3 & 0.36 & 0.45 & 100.6\end{array}$

$\begin{array}{lllll}5.9 & 6.1 & 0.49 & 0.21 & 100.9\end{array}$

$\begin{array}{lllll}6.1 & 6.1 & 0.51 & 0.26 & 100.7\end{array}$

$\begin{array}{lllll}6.1 & 6.1 & 0.50 & 0.25 & 100.7\end{array}$

$\begin{array}{lllll}6.1 & 6.1 & 0.47 & 0.21 & 100.6\end{array}$

$\begin{array}{lllll}6.0 & 6.1 & 0.48 & 0.21 & 100.7\end{array}$

$\begin{array}{lllll}7.1 & 6.0 & 0.63 & 0.36 & 100.0\end{array}$ 
Volcano 5

DTH01-03*

$\begin{array}{llllllllllll}59.5 & 18.5 & 6.0 & 0.0 & 0.34 & 0.21 & 1.0 & 8.9 & 5.3 & 0.12 & 0.11 & 100.0\end{array}$

Volcano 6

DTH02-02*

Volcano 3

DTH05-01*

Volcano 17

$\mathrm{SiO} 2 \mathrm{Al}_{2} \mathrm{O} 3 \mathrm{Fe} 2 \mathrm{O} 3 \mathrm{FeO} \mathrm{MnO} \mathrm{MgO} \mathrm{CaO} \mathrm{Na} 2 \mathrm{O}$ K2O TiO2 P2O5 Total

SO-65 124DS-1n*

$\begin{array}{ll}61.6 & 18.0 \\ & \\ 61.3 & 18.4 \\ & \\ 60.3 & 17.0 \\ & \\ 62.1 & 18.9 \\ 61.8 & 18.8 \\ 60.4 & 18.8 \\ 58.6 & 18.8 \\ 57.1 & 17.7 \\ 60.4 & 18.8 \\ 60.4 & 18.7 \\ 59.2 & 18.9 \\ 60.7 & 19.0 \\ 60.8 & 19.0 \\ 58.9 & 18.3 \\ 58.4 & 18.2 \\ 58.0 & 18.1 \\ 58.4 & 18.2 \\ 58.2 & 18.2\end{array}$

$\begin{array}{lllll}4.0 & 0.0 & 0.18 & 0.81 & 1.3\end{array}$

$\begin{array}{lll}7.2 & 6.0 & 0.59\end{array}$

0.36100 .0

Rocard

PN20-2

PN20-3

PN20-4

PN20-5

PN20-6

PN20-7

PN20-10

PN20-11

PN20-13

PN20-14

PN21-2

PN21-5

PN21-7

PN21-10

PN21-11

$58.2 \quad 18.2$

$\begin{array}{lllll}4.2 & 0.0 & 0.22 & 0.86 & 1.3\end{array}$

$\begin{array}{lllll}6.9 & 6.1 & 0.47 & 0.26 & 100.0\end{array}$

$\begin{array}{lllll}7.0 & 0.0 & 0.46 & 0.21 & 1.2\end{array}$

$\begin{array}{lll}8.4 & 5.1 & 0.13\end{array}$

$0.16 \quad 100.0$

Cyana

SO-47 81DS-3

$59.7 \quad 17.2$

$\begin{array}{llll}4.6 & 0.23 & 0.67 & 1.4\end{array}$

$\begin{array}{lllll}6.1 & 5.7 & 0.48 & 0.26 & 100.8\end{array}$

$\begin{array}{lllllllll}4.6 & 0.24 & 0.72 & 1.5 & 6.1 & 5.8 & 0.50 & 0.27 & 100.6\end{array}$

$\begin{array}{llllllllll}5.0 & 0.22 & 1.29 & 2.0 & 5.7 & 5.8 & 0.84 & 0.48 & 100.7\end{array}$

$\begin{array}{lllllllll}4.6 & 0.18 & 1.18 & 2.0 & 5.8 & 5.9 & 0.79 & 0.45 & 98.6\end{array}$

$\begin{array}{llllllllll}4.6 & 0.22 & 1.16 & 1.9 & 6.5 & 5.6 & 0.50 & 0.48 & 96.2\end{array}$

$\begin{array}{lllllllll}4.9 & 0.19 & 1.30 & 2.0 & 5.6 & 5.8 & 0.85 & 0.51 & 100.7\end{array}$

$\begin{array}{lllllllll}5.0 & 0.23 & 1.29 & 1.9 & 5.9 & 5.8 & 0.84 & 0.49 & 100.9\end{array}$

$\begin{array}{lllllllll}4.8 & 0.23 & 1.26 & 2.0 & 5.7 & 5.9 & 0.80 & 0.48 & 99.6\end{array}$

$\begin{array}{lllllllll}5.0 & 0.20 & 1.29 & 2.0 & 5.7 & 5.8 & 0.81 & 0.50 & 101.4\end{array}$

$\begin{array}{llllllllll}4.6 & 0.20 & 1.07 & 1.9 & 5.8 & 5.7 & 0.79 & 0.42 & 100.6\end{array}$

$\begin{array}{lllllllll}4.8 & 0.18 & 1.34 & 2.1 & 5.2 & 6.2 & 0.91 & 0.34 & 98.6\end{array}$

$\begin{array}{lllllllll}4.8 & 0.17 & 1.35 & 2.1 & 5.1 & 6.1 & 0.87 & 0.35 & 97.7\end{array}$

$\begin{array}{lllllllll}4.7 & 0.16 & 1.27 & 2.0 & 5.4 & 6.2 & 0.86 & 0.32 & 97.2\end{array}$

$\begin{array}{lllllllll}4.8 & 0.17 & 1.29 & 2.0 & 5.2 & 6.1 & 0.85 & 0.31 & 97.6\end{array}$

$\begin{array}{lllllllll}4.8 & 0.19 & 1.29 & 2.0 & 5.1 & 6.1 & 0.85 & 0.32 & 97.4\end{array}$

\section{Large Volcanoes}

Teahitia

PN17-1

PN18-1

$\begin{array}{ll}45.9 & 14.0 \\ 46.1 & 15.5 \\ 50.5 & 17.1\end{array}$

$\begin{array}{llll}2.1 & 0.17 & 6.37 & 12.5\end{array}$

$\begin{array}{llll}2.1 & 0.18 & 4.98 & 10.7\end{array}$

$3.0 \quad 1.6 \quad 4.56$

$0.77 \quad 101.4$

PN19-5

$\begin{array}{llll}9.5 & 0.16 & 3.94 & 8.0\end{array}$

$\begin{array}{lllll}3.7 & 2.4 & 4.53 & 1.00 & 101.6\end{array}$

$\begin{array}{lllll}4.0 & 3.4 & 3.67 & 1.06 & 101.6\end{array}$

* Normalised to $100 \mathrm{wt} . \%$, unpub. data from

Binard doctoral thesis 
Pitcairn

$87 \mathrm{Sr} / 86 \mathrm{Sr} \quad 143 / 144 \mathrm{Nd}$

Small Volcanoes

PNDR1-1

$0.705199 \quad 0.512472$

PNDR3-1

$0.705072 \quad 0.512531$

$0.705078 \quad 0.512525$

PNDR11-2

$0.705195 \quad 0.512485$

PNDR12-1

$0.705306 \quad 0.512452$

PN8-4 